13

\title{
Анализ эмиссии электронов с одиночного кремниевого катода в квазивакуумную (воздушную) среду методом атомно-силовой микроскопии
}

\author{
( И.Д. Евсиков, С.В. Митько, П.Ю. Глаголев, Н.А. Дюжев, Г.Д. Демин
}

Национальный исследовательский университет „МИЭТ“, 124498 Зеленоград, Москва, Россия

e-mail: evsikov.ilija@yandex.ru

Поступило в Редакцию 16 апреля 2020 г.

В окончательной редакции 16 апреля 2020 г.

Принято к публикации 16 апреля 2020 г.

\begin{abstract}
Методом атомно-силовой микроскопии экспериментально рассмотрены особенности полевой эмиссии электронов с одиночного кремниевого катода острийного типа в квазивакуумную (воздушную) среду. В бесконтактном режиме работы атомно-силового микроскопа измерены вольт-амперные характеристики одиночного катода с нанометровым радиусом закругления вершины при расстояниях 10 и $20 \mathrm{~nm}$ между вершиной катода и вершиной измерительного зонда. Проведено моделирование распределения электрических полей как на поверхности вершины одиночного катода, так и на поверхности вершин отдельно взятых катодов в составе массива, на основе которого сделана теоретическая оценка фактора усиления электрического поля в зависимости от расстояния „катод-зонд“. Показано, что фактор усиления поля, рассчитанный из экспериментальных ВАХ в координатах Фаулера-Нордгейма, на несколько порядков превышает величину, полученную из теоретических расчетов, что указывает на необходимость учета дополнительных квантоворазмерных эффектов, играющих важную роль в формировании тока эмиссии электронов в наноразмерном зазоре.
\end{abstract}

Ключевые слова: полевая эмиссия, атомно-силовая микроскопия, кремниевые полевые нанокатоды, коэффициент усиления поля.

DOI: 10.21883/JTF.2020.11.49986.136-20

\section{Введение}

В настоящее время все более широкое развитие в мире приобретает разработка устройств вакуумной наноэлектроники, что связано со стойкостью таких устройств к воздействию радиационного излучения, возможностью работы при высоких температурах, а также их высоким быстродействием (в THz-диапазоне). Данные преимущества являются следствием физических свойств вакуумной среды, которая обеспечивает баллистический перенос носителей заряда (электронов) по каналу проводимости практически без соударений. В свою очередь, фононное рассеяние носителей заряда на кристаллической решетке полупроводников при протекании по ним тока приводит к нежелательному разогреву структуры и возникновению дополнительных дефектов в канале проводимости. Эти негативные эффекты становятся особенно критичными при достижении уровня проектных норм $10 \mathrm{~nm}$ и ниже, что препятствует дальнейшему масштабированию полупроводниковой электроники [1]. Тем не менее сильные стороны приборов вакуумной наноэлектроники (их высокое быстродействие, устойчивость к воздействию радиации и высокой температуры), принцип работы которых основан на эффекте полевой эмиссии электронов в вакуум, могут быть объединены с преимуществами устройств кремниевой КМОП (комплементарная структура металл-оксид-полупро- водник) технологии, что было показано в работах [2-4]. Более того, в возможность подобной интеграции была продемонстрирована ранее в [5] на примере создания комбинированных устройств нового класса, сочетающих в себе стандартный МДП („металл-диэлектрик-полупроводник“) транзистор и вакуумный эмиссионный транзистор с длиной канала проводимости $100 \mathrm{~nm}$ и рабочим напряжением $10 \mathrm{~V}$. Дальнейшее снижение рабочего напряжения и, как следствие, потребляемой мощности, а также повышение срока эксплуатации вакуумных наноразмерных приборов может быть достигнуто путем уменьшения длины канала проводимости [6-9]. Стоит, однако, отметить тот факт, что физика полевой эмиссии электронов на наноразмерном масштабе, когда длина межэлектродного расстояния составляет $20 \mathrm{~nm}$ и ниже, остается все еще слабо изученной, что создает трудности для точного проектирования устройств вакуумной наноэлектроники [10]. В настоящей работе методом атомно-силовой микроскопии (АСМ) проводится измерение вольт-амперных характеристик (BАХ) одиночных кремниевых катодов, эмиссионные параметры которых сравниваются с теоретическими оценками, полученными из моделирования. Поскольку длина свободного пробега электронов в воздухе составляет около $68 \mathrm{~nm}$, данные исследования могут проводиться в условиях квазивакуумной (воздушной) среды без необходимости использования дополнительной вакуумной камеры, что упрощает 
проведение эксперимента. В то же время не требуется вакуумное корпусирование автоэмиссионных устройств с длиной канала менее $20 \mathrm{~nm}$, что делает их совместимыми с ключевыми операциями КМОП- технологии. Исследования эмиссионных структур методом АСМ проводились ранее другими научными группами $[11,12]$. Метод АСМ в данных работах применялся главным образом для получения информации о структуре поверхности исследуемого образца. Отличие нашей работы от вышеупомянутых исследований состоит в экспериментальном рассмотрении полевой эмиссии электронов с кремниевых наноразмерных катодов при суб- $20 \mathrm{~nm}$ квазивакуумном зазоре, что сделано с целью изучения особенностей эффектов полевой эмиссии на данном масштабе. Подобные исследования являются новыми и не проводились ранее.

\section{1. Методика эксперимента}

Исследование процесса полевой эмиссии электронов с одиночного кремниевого катода в квазивакуумный (воздушный) зазор толщиной $20 \mathrm{~nm}$ и ниже проводилось с использованием методик АСМ (рис. 1). В качестве исследуемого образца был взят фрагмент кремниевой пластины n-типа проводимости с имеющимся на нем массивом полевых катодов острийного типа, который был изначально изготовлен для выполнения задач рентгеновской литографии. Каждый из катодов в массиве обеспечивает фиксированную величину автоэмиссионного тока, необходимую для генерации рентгеновского излучения в заданной области анодного электрода с прострельной мишенью, ответственной за создание единичного пикселя изображения на рентгенорезисте [13]. Для успешного формирования топологического рисунка крайне важно иметь однородность распределения автоэмиссионного тока по мишени, что, в свою очередь, требует высокой однородности размера катодов внутри массива. Углеродные наноструктуры (нанотрубки, угле-

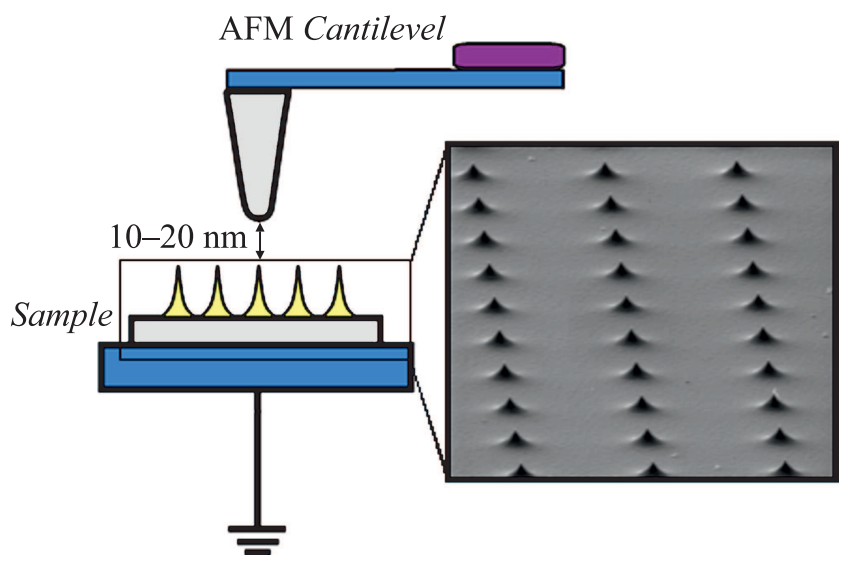

Рис. 1. Схема проведения эксперимента для анализа полевой эмиссии с одиночного полевого катода в квазивакуумную (воздушную) среду с применением метода АСМ. родные монослои (графен) и проч.), проявляющие хорошие автоэмиссионные свойства, не отвечают данному требованию, что связано с особенностью их синтезирования, которое обусловливает низкую воспроизводимость размеров отдельных эмиттеров в составе массива [14]. Как следствие, это приводит к неконтролируемому возникновению и „выгоранию,эмиссионных центров, а также заметному разбросу значений автоэмиссионного тока эмиттеров по массиву [15]. Подобная проблема возникает и в технологии создания автоэмиссионных катодов Спиндта на основе тугоплавких металлов (Au, $\mathrm{W}, \mathrm{Mo})$, где травление металлических слоев является сложной технологической процедурой и не позволяет достичь близких значений радиусов их вершин [16]. В работе [17] были показаны исключительные преимущества традиционной кремниевой технологии для создания воспроизводимых автоэмиссионных структур разброс размеров катодов внутри массива оказывается минимальным, а значения плотности тока могут превышать $100 \mathrm{~A} / \mathrm{cm}^{2}$ при напряжении менее $75 \mathrm{~V}$. Достаточно высокая воспроизводимость технологического процесса создания массива кремниевых катодов острийного типа позволяет формировать структуры, обладающие строгой периодичностью, что особенно важно для реализации устройств, требующих генерации направленных электронных пучков высокой плотности - в частности, системы микрофокусных рентгеновских источников. Технологический процесс создания массива кремниевых катодов острийного типа для указанного применения был описан нами ранее в $[13,17]$. Предварительное исследование образцов методикой РЭМ (растровой электронной микроскопии) позволило сделать вывод о том, что полученные катоды обладают наноразмерным (порядка $10 \mathrm{~nm}$ ) радиусом скругления вершины и высотой около $350 \mathrm{~nm}$.

Измерения ВАХ одиночного кремниевого катода из массива проводились с помощью атомно-силового микроскопа марки SmartSPM-SPM 1000 фирмы AIST-NT (рис. 2). При проведении измерений использовался кантилевер K-TEK Nano DCP20 с алмазным покрытием и радиусом закругления зонда $35 \mathrm{~nm}$. Приборный столик с образцом во время измерений имел нулевой потенциал. Измерение ВАХ отдельно взятых катодов проводилось в двух режимах сканирования поверхности образца: контактном и бесконтактном. На кантилевер атомносилового микроскопа подавалось переменное напряжение с частотой, близкой к собственной частоте колебаний кантилевера (порядка десятков kHz). Предварительный подогрев образца не производился. Особенностью проведения измерений эмиссионных характеристик образца методом АСМ является то, что сканирование поверхности образца можно производить на расстоянии менее $20 \mathrm{~nm}$ от вершины выбранного острийного катода, а сам процесс полевой эмиссии инициировать при атмосферном давлении. Поскольку длина свободного пробега электронов в воздухе составляет около $68 \mathrm{~nm}$, при заданных расстояниях (от $20 \mathrm{~nm} \mathrm{и} \mathrm{ниже)}$ 


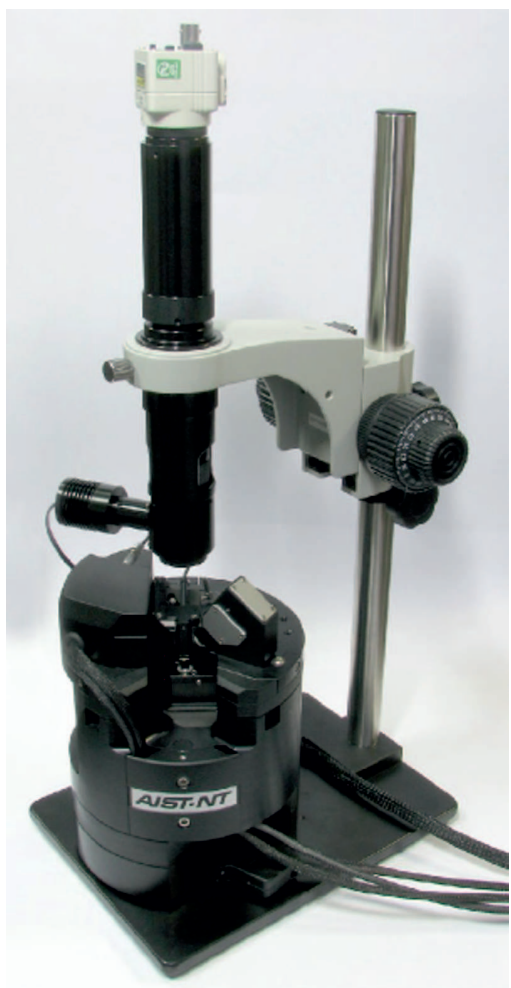

Рис. 2. Внешний вид атомно-силового „SMART-SPM 1000“, используемого для эксперимента.

между образцом и измерительным зондом атомно-силового микроскопа вероятность столкновения электронов с молекулами газов среды оказывается пренебрежимо малой [18]. По этой причине воздушную среду, в которой производятся измерения, можно считать эквивалентной вакууму.

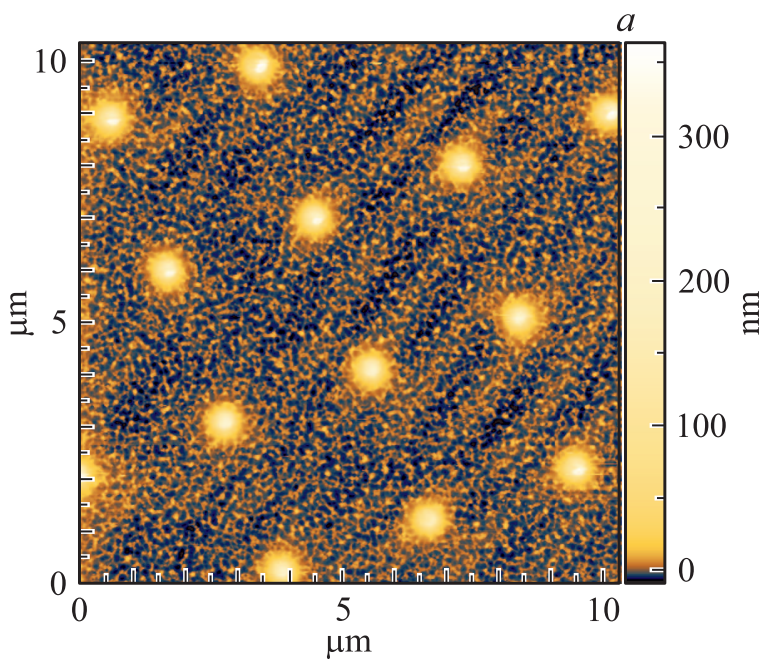

\section{2. Результаты эксперимента}

На рис. 3, $a$ приведено изображение отсканированного рельефа фрагмента образца со шкалой высот. По ярким периодическим областям на изображении можно идентифицировать исследуемые полевые катоды. В свою очередь, рис. $3, b$ представляет собой трехмерное изображение фрагмента массива исследуемых острийных полевых катодов, полученное непосредственно с атомно-силового микроскопа бесконтактным методом сканирования.

Два основных параметра, характеризующих эффективность работы полевых катодов - это коэффициент усиления поля $\beta$ и эффективная площадь эмиссии $S_{\text {eff. }}$ Как известно, эмиссионный ток полевого катода напрямую зависит от напряженности локального электрического поля $E_{S}$ на поверхности вершины этого катода. Обычно напряженность локального поля $E_{S}$ значительно превосходит напряженность „макроскопического,поля $E_{m}$, справедливую для случая плоскопараллельной конфигурации электродов. Отношение напряженностей указанных полей описывается коэффициентом усиления поля $\beta[19]$ :

$$
\beta=\frac{E_{S}}{E_{m}}=E_{S} \frac{d}{U},
$$

где $d$ - расстояние „катод-измерительный зонд“, $U$ межэлектродное напряжение (между вершиной катода и вершиной зонда кантилевера).

Эффективная площадь эмиссии $S_{\text {eff }}$ в общем случае, т.е. при условии, что эмиттер обладает идеальной геометрией, а локальное электрическое поле $E_{S}$ на поверхности эмиттера и работа выхода эмиттера $\varphi$ однородны, формально выражается как $S_{\mathrm{eff}}=I_{f e} / j_{f e}$, где $I_{f e}-$ полный эмиссионный ток катода, $j_{f e}-$ плотность тока эмиссии. При неоднородном распределении поля по поверхности катода полный ток с эмиттера рассчитывается через интегрирование плотности тока

Рис. 3. $a$ - АСМ-изображение фрагмента массива кремниевых катодов (вид сверху); $b$ - трехмерная модель фрагмента массива кремниевых катодов, полученная с использованием АСМ. 
$a$

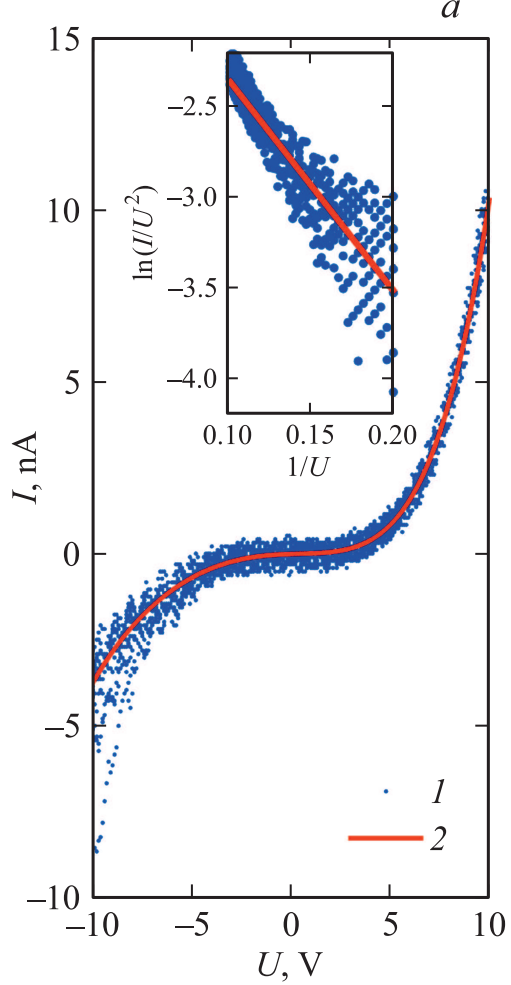

$b$

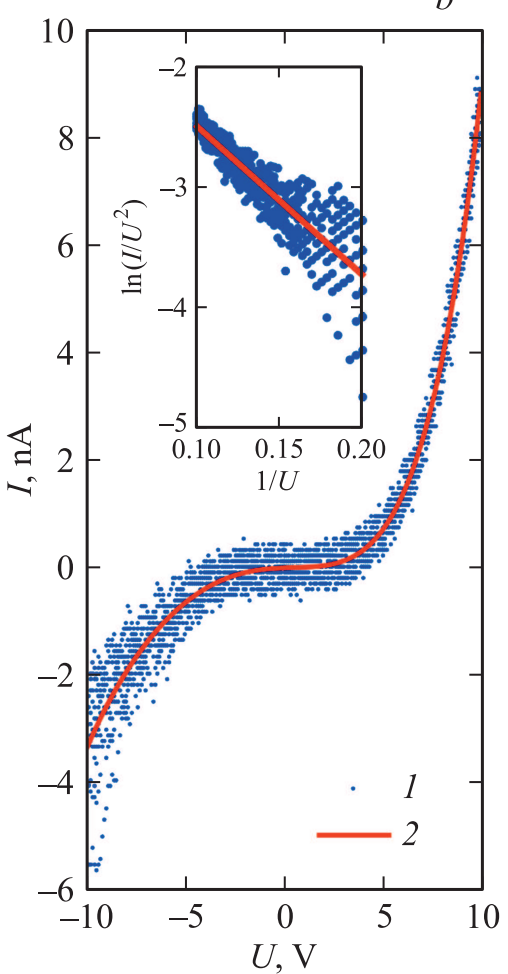

C

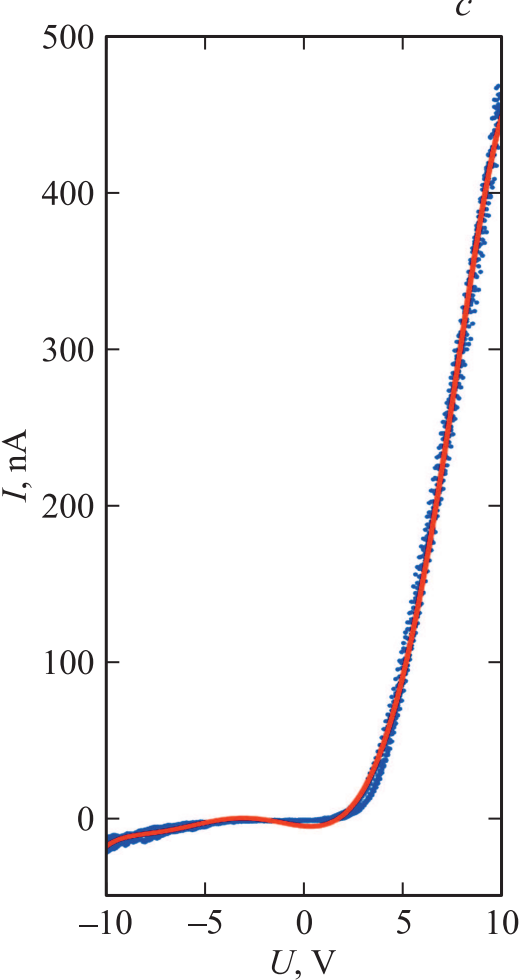

Рис. 4. ВАХ, снятые с одиночного кремниевого катода в обычных координатах и в координатах Фаулера-Нордгейма (см. вставку) при: $a$ - расстоянии катод-зонд $10 \mathrm{~nm}, b-$ расстоянии катод-зонд $20 \mathrm{~nm}, c-$ контакте зонда с катодом. $1-$ экспериментальные кривые, 2 - аппроксимирующие кривые.

по его поверхности: $I_{f e}=\int j_{f e}\left(E_{S}\right) d S$. При экспериментальном исследовании реальных систем „катод-анод“ для определения вышеназванных эффективных параметров ( $\beta$ и $\left.S_{\text {eff }}\right)$ предпочтительнее использовать метод анализа ВАХ эмиссионной структуры, построенных в полулогарифмических координатах Фаулера-Нордгейма и аппроксимированных линейной зависимостью. Эффективные параметры связаны с наклоном $(K)$ и отсечкой $(R)$ линии тренда, которые, в свою очередь, могут быть получены из уравнения полевой эмиссии, приведенного к макроскопическим величинам тока $I_{f e}$ и напряжения $U[20]$ :

$$
I_{f e}=S_{\mathrm{eff}} A\left(\frac{\beta U}{d \varphi^{1 / 2}}\right)^{2} \exp \left(-\frac{B d \varphi^{3 / 2}}{\beta U}\right)
$$

где $\varphi$ - работа выхода материала катода, $A=$ $=1.54 \cdot 10^{-6}\left[\mathrm{~A} \cdot \mathrm{eV} \cdot \mathrm{V}^{-2}\right]-$ первая константа Фауле$\mathrm{pa}-$ Нордгейма, $B=6.83 \cdot 10^{9}\left[\mathrm{eV}^{-3 / 2} \cdot \mathrm{V} \cdot \mathrm{m}^{-1}\right]-$ вторая константа Фаулера-Нордгейма. В координатах Фаулера-Нордгейма уравнение (2) принимает соответствующий вид

$$
\ln \left(\frac{I_{f e}}{U^{2}}\right)=\ln \left(\frac{A S_{\mathrm{eff}} \beta^{2}}{d^{2} \varphi}\right)-\frac{B d \varphi^{3 / 2}}{\beta U}=R+\frac{K}{U}
$$

Согласно уравнению (3), эффективные параметры эмиттера рассчитываются как

$$
\beta=\frac{B d}{K}, \quad S_{\text {eff }}=\frac{\exp (R) d^{2}}{A \beta^{2}} .
$$

На рис. 4 приведены три типа ВАХ одиночного кремниевого катода, полученные путем вариации напряжения в диапазоне от -10 до $10 \mathrm{~V}$, которое подавалось на кантилевер атомно-силового микроскопа, как в случае бесконтактного (рис. $4, a-$ для $d=10 \mathrm{~nm}$, рис. $4, b-$ для $d=20 \mathrm{~nm}$ ), так и в случае контактного (рис. 4,c - при непосредственном контакте зонда с катодом) режима сканирования. Вариация напряжения была произведена пять раз подряд без продолжительных временных интервалов между „проходами“. На вставках к рис. 4 приведены экспериментальные и линейные аппроксимирующие зависимости ВАХ полевого катода, построенные в координатах Фаулера-Нордгейма при расстоянии между катодом и зондом кантилевера, равном 10 и $20 \mathrm{~nm}$ соответственно. Значения ВАХ в координатах Фаулера-Нордгейма приведены для вариации напряжения в диапазоне от 5 до $10 \mathrm{~V}$, когда ток катода достигает значений выше $1 \mathrm{nA}$ (участок стабильной полевой эмиссии). Кривые, аппроксимирующие ВАХ в стандартных координатах для соответствующих расстояний „катод-измерительный зонд“, равных 10 и $20 \mathrm{~nm}$, могут 
быть описаны в форме суммы экспоненциальных зависимостей вида $I_{f e}(U)=a_{1} \cdot \exp \left(b_{1} U\right)-a_{2} \cdot \exp \left(-b_{2} U\right)$, где аппроксимирующие коэффициенты $a_{1}=9.754 \cdot 10^{-2}$, $b_{1}=4.719 \cdot 10^{-1}, \quad a_{2}=1.317 \cdot 10^{-1}, \quad b_{2}=3.599 \cdot 10^{-1}$ для ВАХ на рис. $4, a(d=10 \mathrm{~nm})$ и $a_{1}=8.469 \cdot 10^{-2}$, $b_{1}=4.7 \cdot 10^{-1}, a_{2}=1.343 \cdot 10^{-1}, b_{2}=3.279 \cdot 10^{-1}$ для $\mathrm{BAX}$ на рис. $4, b(d=20 \mathrm{~nm})$. Форма аппроксимирующих зависимостей $I_{f e}(U)$ из рис. $4, a$ и $b$ определяется тем, что ВАХ для заданных расстояний „катод-измерительный зонд“ (10 и $20 \mathrm{~nm}$ соответственно) можно подразделить на два участка - участок с положительным $(U>0)$ и участок с отрицательным $(U<0)$ напряжением, подаваемым на кантилевер АСМ. В случае $U>0$ полевая эмиссия электронов происходит с одиночного кремниевого катода на измерительный зонд АCM, в обратном случае (при $U<0)$ - с измерительного зонда на катод. Стоит отметить, что эмиссионные свойства зонда кантилевера отличаются от эмиссионных свойств кремниевого катода, что объясняет асимметричный характер ВАХ относительно нуля. В свою очередь, кривая, аппроксимирующая BAX в стандартных координатах в случае контакта катода с измерительным зондом (рис. 4,c), представляет собой полином 6-ой степени вида $I_{f e}=f(U)=\sum_{i=0.6} C_{i} U^{i}$, где $C_{0}=-3.633$, $C_{1}=-1.071, C_{2}=1.316, C_{3}=4.789 \cdot 10^{-1}, C_{4}=3.206 \cdot 10^{-2}$, $C_{5}=-2.348 \cdot 10^{-4}, C_{6}=-2.32 \cdot 10^{-4}$. Коэффициенты детерминации $R^{2}$, которые характеризуют величину достоверности аппроксимации соответствующих $\mathrm{BAX}$ на рис. 4, построенных в обычных координатах, равны: $R^{2}=0.9636$ (рис. 4, $a$ ), $R^{2}=0.9661$ (рис. 4,b) и $R^{2}=$ $=0.9966$ (рис. 4,c). Линейная зависимость, аппроксимирующая экспериментальные ВАХ, построенные в координатах Фаулера-Нордгейма, имеет вид $\ln \left(I_{f e} / U^{2}\right)=$ $=a+b / U$, где $a=-1.175, b=-11.75$ для $\mathrm{BAX}$ на вставке к рис. $4, a, a=-1.231, b=-12.56$ для $\mathrm{BAX}$ на вставке к рис. $4, b$. Коэффициенты детерминации $R^{2}$ соответствующих ВАХ на вставках к рисункам равны: $R^{2}=0.8278$ (рис. $\left.4, a\right), R^{2}=0.7969$ (рис. $4, b$ ).

В таблице приведены рассчитанные по формуле (4) эффективные параметры эмиссии одиночного кремниевого катода для двух расстояний $d=10 \mathrm{~nm}$ и $d=20 \mathrm{~nm}$, выбранных в эксперименте. Как видно из таблицы, коэффициент усиления поля $\beta$ возрастает пропорционально расстоянию от зонда до исследуемого катода. В свою очередь, как показывают расчеты, площадь эмис-

Эффективные параметры одиночного кремниевого катода для различных расстояний между катодом и измерительным зондом

\begin{tabular}{c|r|c}
\hline Расстояние „катод-зонд“, $\mathrm{nm}$ & $\beta$ & $S_{\text {eff, }} \mu \mathrm{m}^{2}$ \\
\hline 10 & 55.5 & 0.318 \\
20 & 103.8 & 0.343
\end{tabular}

сии $S_{\text {eff }}$ при увеличении расстояния „катод-измерительный зонд“ возросла незначительно - в пределах 8\%.

\section{3. Модель и основные уравнения}

Для сравнения экспериментальных и теоретических результатов в рамках настоящей работы методом конечных элементов было проведено численное моделирование распределения электрического поля по поверхности образца кремниевого катода, исследуемого методом АСМ. Для этой цели с помощью программного пакета COMSOL MultiPhysics [21] были созданы две трехмерные модели, воспроизводящие проведение эксперимента и представляющие собой следующие системы: 1) „одиночный катод-измерительный зонд“ и 2) „массив катодов-измерительный зонд,. Результаты исследования образцов с помощью методик РЭМ и АСМ были использованы для определения величин геометрических параметров катодов (высота, радиус скругления вершины, форма поверхности катода).

Для расчета распределения электрического поля по поверхности вершины одиночного кремниевого катода и по поверхности вершин катодов в составе массива был использован физико-математический модуль „Electrostatics“ в составе программного пакета COMSOL MultiPhysics, где напряженность поля $\mathbf{E}=-\nabla \phi$ рассчитывается из решения уравнения Пуассона:

$$
-\nabla \cdot(\nabla \phi)=\frac{\rho}{\varepsilon_{0}}
$$

где $\phi=\phi(\mathbf{r})$ - потенциал в произвольной точке $\mathbf{r}=(x, y, z)$ системы, $\rho$ - объемная плотность заряда, $\varepsilon_{0}=8.85 \cdot 10^{-12}\left[\mathrm{~F} \cdot \mathrm{m}^{-1}\right]$ - диэлектрическая проницаемость вакуума. В общем случае с учетом диэлектрических свойств материалов уравнение (5) принимает следующий вид: $\nabla \cdot\left(\varepsilon_{0} \varepsilon_{r} \mathbf{E}\right)$, где $\varepsilon_{r}-$ тензор диэлектрической проницаемости 3-го ранга. Расчет коэффициента усиления поля $\beta$ на вершине катода производился по формуле (1).

\section{4. Результаты моделирования}

Рис. 5 иллюстрирует результаты моделирования распределения электрического поля по поверхности вершины одиночного кремниевого катода для $d=10 \mathrm{~nm}$, и приложенное напряжение $U$ составляет $10 \mathrm{~V}$, что соответствует эксперименту. На вставке к рисунку представлена зависимость $\beta$ вдоль линии $L$ пересечения вертикальной плоскостью поверхности катода, демонстрирующая тенденцию экспоненциального уменьшения коэффициента усиления поля по мере удаления от вершины кремниевого эмиттера. При заданном расстоянии $d=10 \mathrm{~nm}$ рассчитанное значение коэффициента $\beta$ усиления поля на вершине одиночного катода составляет около 2.25, что на порядок ниже значения, полученного нами ранее из экспериментальных ВАХ 


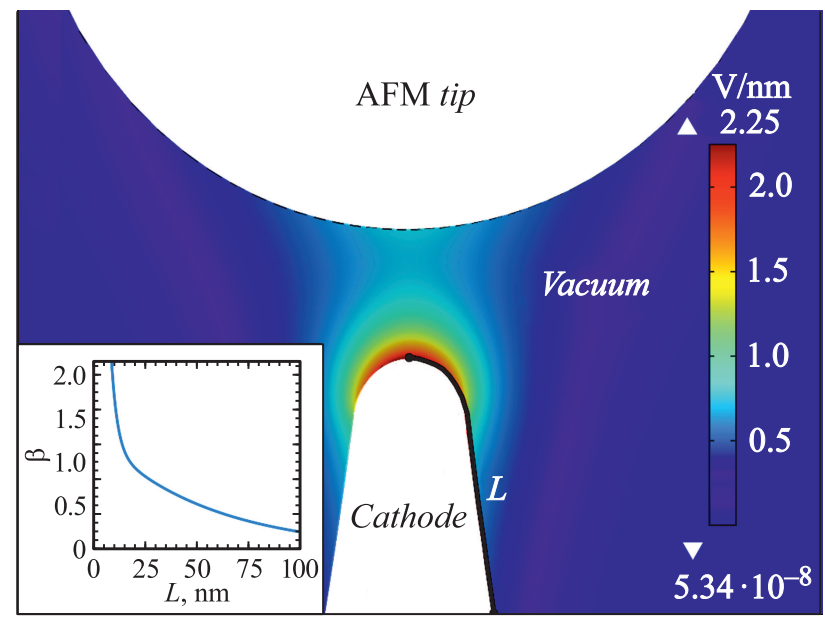

Рис. 5. Распределение электрического поля в системе „одиночный катод-измерительный зонд кантилевера“ при фиксированном расстоянии $d$ „катод-измерительный зонд“, равном $10 \mathrm{~nm}$, и напряжении $U=10 \mathrm{~V}$. На вставке к рисунку - изменение амплитуды электрического поля вдоль линии $L$ среза пересечения поверхности катода вертикальной плоскостью в зависимости от расстояния от его вершины.

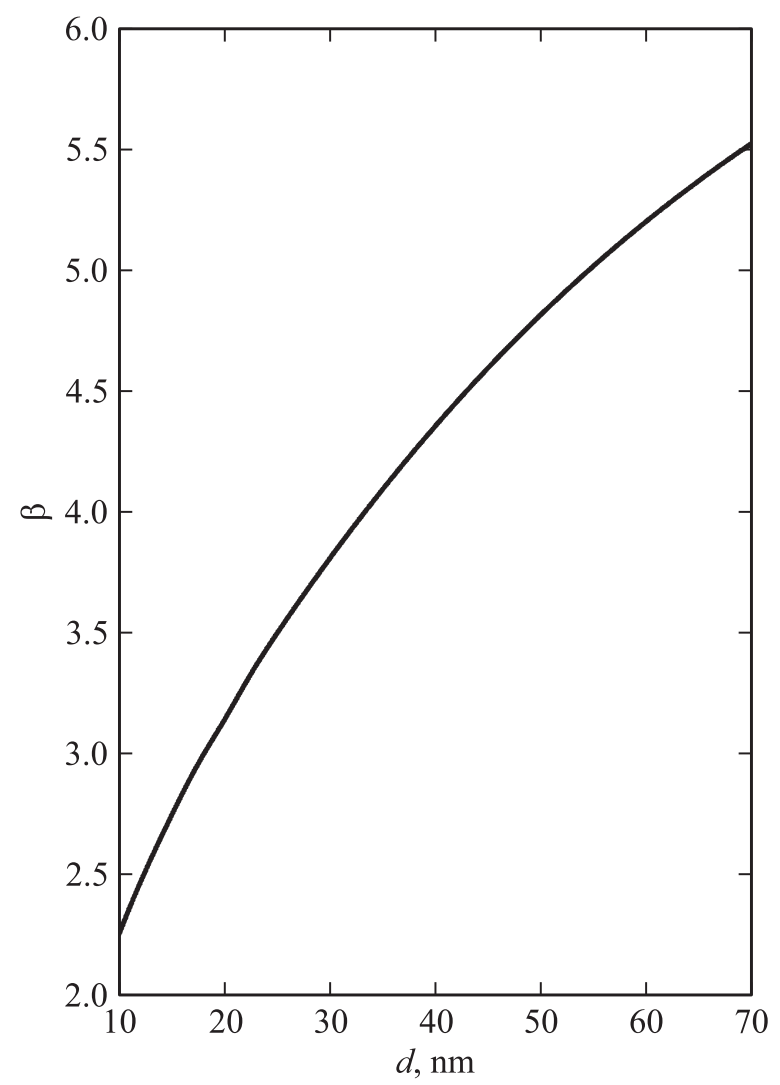

Pис. 6. Коэффициент усиления электрического поля $\beta$ на вершине одиночного кремниевого катода как функция от расстояния $d$ „катод-измерительный зонд“.

одиночного катода. Данный факт может быть обусловлен изменением формы („вытягиванием“ отдельных участ- ков) поверхности кремниевого эмиттера под действием сильного электрического поля в нанометровом зазоре, что наблюдалось ранее в [22] на расстояниях „катод-зонд“ в несколько сотен нанометров. Подобный эффект может обусловливать искажение потенциального барьера на границе „кремний-вакуум“, который зависит от радиуса кривизны поверхности кремниевого эмиттера $[23,24]$ и, как следствие, изменение фактора $\beta$ и величины автоэмиссионного тока, что может объяснять расхождение с экспериментом и что следует учитывать в процессе моделирования. На рис. 6 приведен график зависимости коэффициента усиления поля $\beta$ от расстояния „катод-измерительный зонд“, варьируемого в диапазоне от 10 до $70 \mathrm{~nm}$ (в пределах длины свободного пробега электронов в воздухе). Как видно из рисунка, коэффициент $\beta$ плавно возрастает с расстоянием $d$, что также наблюдается в эксперименте и связано с более быстрой динамикой уменьшения величины „макроскопического“ поля $E_{m}=U / d$ по отношению к падению локального поля $E_{S}$ на вершине катода. Зависимость $\beta=f(d)$ идеально (коэффициент детерминации $R^{2}=1$ ) аппроксимируется аналитической функцией $\beta=b_{0}+b_{1} d+b_{2} / d+b_{3} / d^{2}$, где $b_{0}=2.074$, $b_{1}=0.07383, b_{2}=-5.362, b_{3}=-3.349 \cdot 10^{-4}$. Результаты моделирования электрического поля для случая матрицы катодов размерностью $3 \times 3$ демонстрируют однородное распределение поля по вершинам катодов (рис. 7), величина которого согласуется с ранее рассчитанной для одиночного кремниевого катода. В силу того, что расстояние между катодами в экспериментальной матрице составляет около $3 \mu \mathrm{m}$, окружение „выбранного“ зондом катода не значительно сказывается на изменении электрического поля на его поверхности.

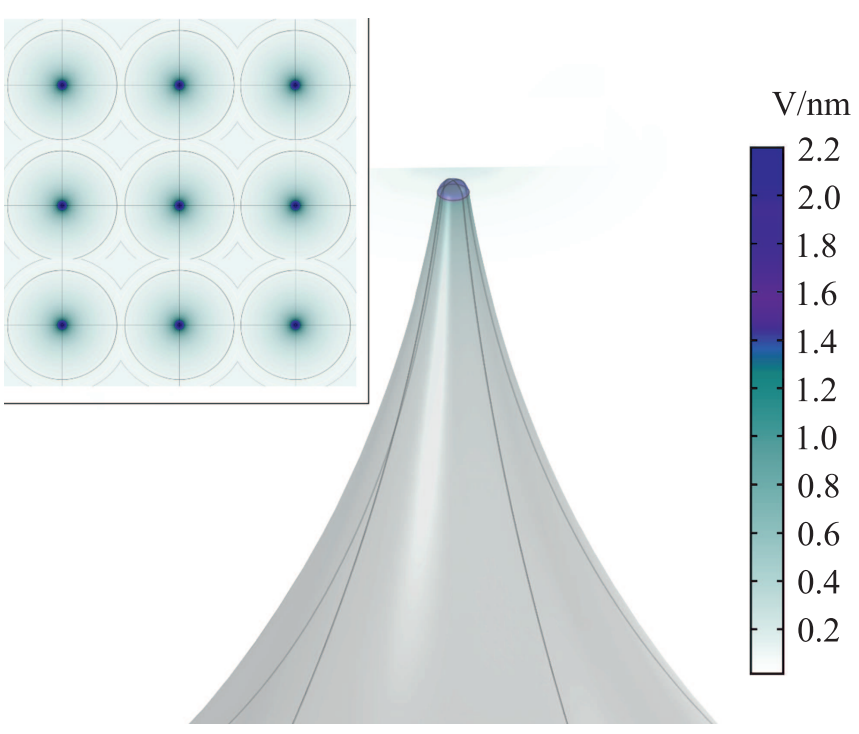

Рис. 7. Распределение электрического поля в системе „массив катодов-измерительный зонд кантилевера“. Размерность массива катодов $-3 \times 3$, расстояние $d=10 \mathrm{~nm}$, приложенное к зонду напряжение $U=10 \mathrm{~V}$. На вставке - распределение электрического поля всего массива катодов (вид сверху). 


\section{Заключение}

Таким образом, в работе рассмотрен метод анализа полевой эмиссии с одиночного кремниевого катода в воздушной среде (квазивакууме) на наноразмерном масштабе. Методом АСМ измерены ВАХ одиночного кремниевого катода в составе катодной матрицы, позволяющие судить об особенностях полевой эмиссии на соответствующей длине квазивакуумного канала проводимости - 10 и $20 \mathrm{~nm}$. Для каждого случая рассчитаны эффективные параметры эмиссии - коэффициент усиления поля $\beta$ и эффективная площадь эмиссии $S_{\text {eff. Для }}$ объяснения результатов эксперимента было проведено моделирование электрического поля в рассматриваемой системе „образец-зонд АСМ“, где в качестве образца был рассмотрен как одиночный острийный катод, так и массив острийных катодов размерности $3 \times 3$. Анализ полученных результатов позволяет сделать вывод о необходимости учета дополнительных эффектов, возникающих вблизи поверхности эмиттера, в частности искажения потенциального барьера вследствие вероятной деформации приповерхностной области эмиттера под действием сильного электрического поля в наноразмерном зазоре, что может объяснить расхождение полученных теоретических оценок фактора усиления поля с экспериментом. Полученные результаты могут быть применимы для разработки корректного теоретического описания автоэлектронной эмиссии с полупроводниковых эмиттеров в наномасштабе, находящегося за пределами классической теории Фаулера-Нордгейма.

\section{Финансирование работы}

Работа выполнена при финансовой поддержке гранта президента РФ (№ 075-15- 2019-1139) с использованием оборудования ЦКП „Микросистемная техника и электронная компонентная база“, поддержанного Минобрнауки России.

\section{Конфликт интересов}

Авторы заявляют, что у них нет конфликта интересов.

\section{Список литературы}

[1] Seshan $K$. in Handbook of Thin Film Deposition. Elsevier, 2018. P. 19-41. DOI: 10.1016/B978-0-12-812311-9.00002-5

[2] Nirantar S., Ahmed T., Bhaskaran M., Han J.-W., Walia S., Sriram S. // Adv. Intel. Systems. 2019. Vol. 1. P. 1900039. DOI: $10.1002 /$ aisy.201900039

[3] Han J.-W., Moon D.-I., Meyyappan M. // Nano Lett. 2017. Vol. 17. P. 2146. DOI: 10.1021 /acs.nanolett.6b04363

[4] Han J.-W., Seol M.-L., Moon D.-L., Hunter G., Meyyappan M. // Nat. Electron. 2019. Vol. 2. P. 405. DOI: $10.1038 / \mathrm{s} 41928-019-0289-\mathrm{z}$

[5] Han J.-W., Oh J.S., Meyyappan M. // IEEE Trans. Nanotech. 2014. Vol. 13. P. 464. DOI: 10.1109/TNANO.2014.2310774
[6] Nirantar S., Ahmed T., Ren G., Gutruf P., Xu C., Bhaskaran M., Walia S., Sriram S. // Nano Lett. 2018. Vol. 18. P. 7478. DOI: 10.1021 /acs.nanolett.8b02849

[7] Xu J., Wang Q., Tao Z., Zhai Y., Guangdian C., Qi Z., Zhang X. // IEEE Trans. Electron Dev. 2017. Vol. 64. P. 2364. DOI: 10.1109/TED.2017.2673853

[8] Xu J., Hu H., Yang W., Li C., Shi Y., Shi Y., Wang Q., Zhang $X$. // Nanotechnology. 2020. Vol. 31. P. 065202. DOI: $10.1088 / 1361-6528 / \mathrm{ab} 51 \mathrm{cb}$

[9] Liu M., Fu W., Yang Y., Li T., Wang Y. // Appl. Phys. Lett. 2018. Vol. 112. P. 093104. DOI: $10.1063 / 1.4996370$

[10] Chang W.-T., Hsu H.-J., Pao P.-H. // Micromachines. 2019. Vol. 10. P. 858. DOI: $10.3390 / \mathrm{mi} 10120858$

[11] Chatterjee V., Harniman R., May P.W., Barhai P.K. // Appl. Phys. Lett. 2014. Vol. 104. P. 171907. DOI: $10.1063 / 1.4875059$

[12] Конакова Р.В., Охрименко О.Б., Светличный А.М., Агеев О.А., Волков Е.Ю., Коломийцев А.С., Житяев И.Л., Спиридонов О.Б. // ФТП. 2015. Т. 49. Вып. 9. C. 1278-1281. [Konakova R.V., Okhrimenko O.B., Svetlichnyi A.M., Ageev O.A., Volkov E. Yu., Kolomiytsev A.S., Jityaev I.L., Spiridonov O.B. // Semiconductors. 2015. Vol. 49. N 9. P. $1242-1245$. DOI: $10.1134 / \mathrm{S} 1063782615090146]$

[13] Дюжев Н.А., Демин Г.Д., Филиппов Н.А., Евсиков И.Д., Глаголев П.Ю., Махиборода М.А., Чхало Н.И., Салащенко Н.Н., Филиппов С.В., Колосько А.Г., Попов Е.О., Беспалов В.А. // ЖТФ. 2019. Т. 89. Вып.12. C. 1836-1842. [Djuzhev N.A., Demin G.D., Filippov N.A., Evsikov I.D., Glagolev P.Y., Makhiboroda M.A., Chkhalo N.I., Salashchenko N.N., Filippov S.V., Kolosko A.G., Popov E.O., Bespalov V.A. // Tech. Phys. 2019. Vol. 64. N 12. P. 1742.] DOI: $10.1134 / \mathrm{S} 1063784219120053$

[14] Jung S.M., Hahn J., Jung H.Y., Suh J.S. // Nano Lett. 2006. Vol. 6. P. $1569-1573$. DOI: $10.1021 / \mathrm{nl} 060437 \mathrm{q}$

[15] Zhu N., Chen J. // Micromachines. 2017. Vol. 8. P. 162. DOI: $10.3390 / \mathrm{mi} 8050162$

[16] Guerrera S.A., Akinwande A.I. // Nanotechnology. 2016. Vol. 27. P. 295302. DOI: $10.1088 / 0957-4484 / 27 / 29 / 295302$

[17] Demin G.D., Djuzhev N.A., Filippov N.A., Glagolev P.Yu., Evsikov I.D., Patyukov N.N. // J. Vac. Sci. Technol. B. 2019. Vol. 37. P. 022903. DOI: $10.1116 / 1.5068688$

[18] Qian L., Wang Y., Liu L., Fan S. // J. Vac. Sci. Technol. B. 2010. Vol. 28. P. 562. DOI: $10.1116 / 1.3372333$

[19] Forbes R.G., Edgcombe C.J., Valdré U. // Ultramicroscopy. 2003. Vol. 95. P. 57. DOI: 10.1016/s0304-3991(02)00297-8

[20] Попов Е.О., Колосько А.Г., Чумак М.А., Филиппов С.В. // ЖТФ. 2019. Т. 89. Вып. 10. С. 1615-1625. [Popov E.O., Kolosko A.G., Chumak M.A., Filippov S.V. // Tech. Phys. 2019. Vol. 64. N 10. P. 1530. DOI: 10.1134/S1063784219100177]

[21] COMSOL Multiphysics. Vol. 5.5. COMSOL AB, Stockholm, Sweden, https://www.comsol.com/

[22] Nguyen H.D., Kang J.S., Li M., Hu Y. // Nanoscale. 2019. Vol. 11. P. 3129. DOI: $10.1039 / \mathrm{c} 8 \mathrm{nr} 07912 \mathrm{a}$

[23] Huang Y., Deng Z., Wang W., Liang C., She J., Deng S., $X u$ N. // Sci. Rep. 2015. Vol. 5. P. 10631. DOI: $10.1038 /$ srep10631

[24] Driscoll J.A., Bubin S., French W.R., Varga K. // Nanotechnology. 2011. Vol. 22. P. 285702. DOI: $10.1088 / 0957-4484 / 22 / 28 / 285702$ 